\title{
The precision of the IACT mechanical mounts of the TAIGA observatory
}

\author{
A. Borodin, ${ }^{a, *}$ A. Grinyuk, ${ }^{a}$ A. Pan, ${ }^{a, c}$ Y. Sagan, ${ }^{a, b}$ L. Tkachev ${ }^{a, b}$ and D. Zhurov ${ }^{d}$ on \\ behalf of the TAIGA Collaboration \\ (a complete list of authors can be found at the end of the proceedings)
}

a Joint Institute for Nuclear Research

141980, Dubna, Russian Federation

${ }^{b}$ Dubna State University

141980, Dubna, Russian Federation

${ }^{c}$ The Institute of Nuclear Physics, Ministry of Energy of the Republic of Kazakhstan 050032, Almaty, Kazakhstan

${ }^{d}$ Irkutsk State University

664003 , Irkutsk, Russian Federation

E-mail: artur_b@mail.ru

The TAIGA (Tunka Advanced Instrument for cosmic ray physics and Gamma Astronomy) observatory is located in the Tunka valley ( $\sim 50 \mathrm{~km}$ west from the southern shore of Lake Baikal) at an altitude of $675 \mathrm{~m}$ a.s.1. The TAIGA observatory aims to address gamma-ray astronomy at energies from a few $\mathrm{TeV}$ to several $\mathrm{PeV}$ and $\mathrm{CR}$ physics from $100 \mathrm{TeV}$ to several $\mathrm{EeV}$. Its main feature is the complementary, hybrid approach to distinguish CR events from those of gamma rays. Currently TAIGA consists of $\sim 80$ wide-angle air Cherenkov detectors (HiSCORE stations), three $\sim 4 \mathrm{~m}$ diameter IACTs and several hundred surface and underground muon detectors, grouped in three jointly operating arrays. The exceptional feature of the TAIGA IACT array is it's topology that allows one to aim for the optimal cost/performance by scanning the optimal inter-telescope distances from $300 \mathrm{~m}$ up to $600 \mathrm{~m}$. The IACTs have alt-azimuth type mounts and 576-pixel imaging cameras in the foci, covering $9.6^{\circ}$ aperture in the sky. The segmented reflectors of $\sim 10$ $\mathrm{m}^{2}$ area follow the Davis-Cotton design. The largest diameter of the hexagonal shape reflector is $4.3 \mathrm{~m}$ and the focal length is $4.75 \mathrm{~m}$. The rigid telescope mount provides a maximum displacement of EAS image below $2 \mathrm{~mm}$ (i.e. $\leq 0.024^{\circ}$ ) in the photodetector plane. The main parameters of IACTs are of a crucial importance for their efficient operation and is presented

$37^{\text {th }}$ International Cosmic Ray Conference (ICRC 2021)

July 12 th - 23rd, 2021

Online - Berlin, Germany

\footnotetext{
${ }^{*}$ Presenter
} 


\section{Introduction. TAIGA observatory.}

Up to now majority of information about fluxes and sources of high energy gamma rays was obtained at the following experiments: HEGRA[1], H.E.S.S.[2], MAGIC[3] and VERITAS[4]. They consist of arrays of several telescopes - detectors of Cherenkov radiation from Extensive Air Showers (EAS). Telescopes at a distance $\sim 100 \mathrm{~m}$ from each other have focusing mirrors that reflect Cherenkov light to the camera made out of photomultipliers so that an image of EAS is formed.

One of priority goals of TAIGA observatory (Tunka Advanced Instrument for cosmic ray physics and Gamma Astronomy) is investigation of gamma rays in energy region from several $\mathrm{TeV}$ to $1 \mathrm{PeV}$, which requires extension of experimental area up to several square $\mathrm{km}$. TAIGA observatory distinguishing feature is in combination of measurement of EAS in Imaging Atmospheric Cherenkov Telescope (TAIGA-IACT) and in an array of wide-angle Cherenkov detectors (TAIGA-HiSCORE)[5]. At present time 2 IACTs are operating as part of TAIGA observatory (Fig.1).

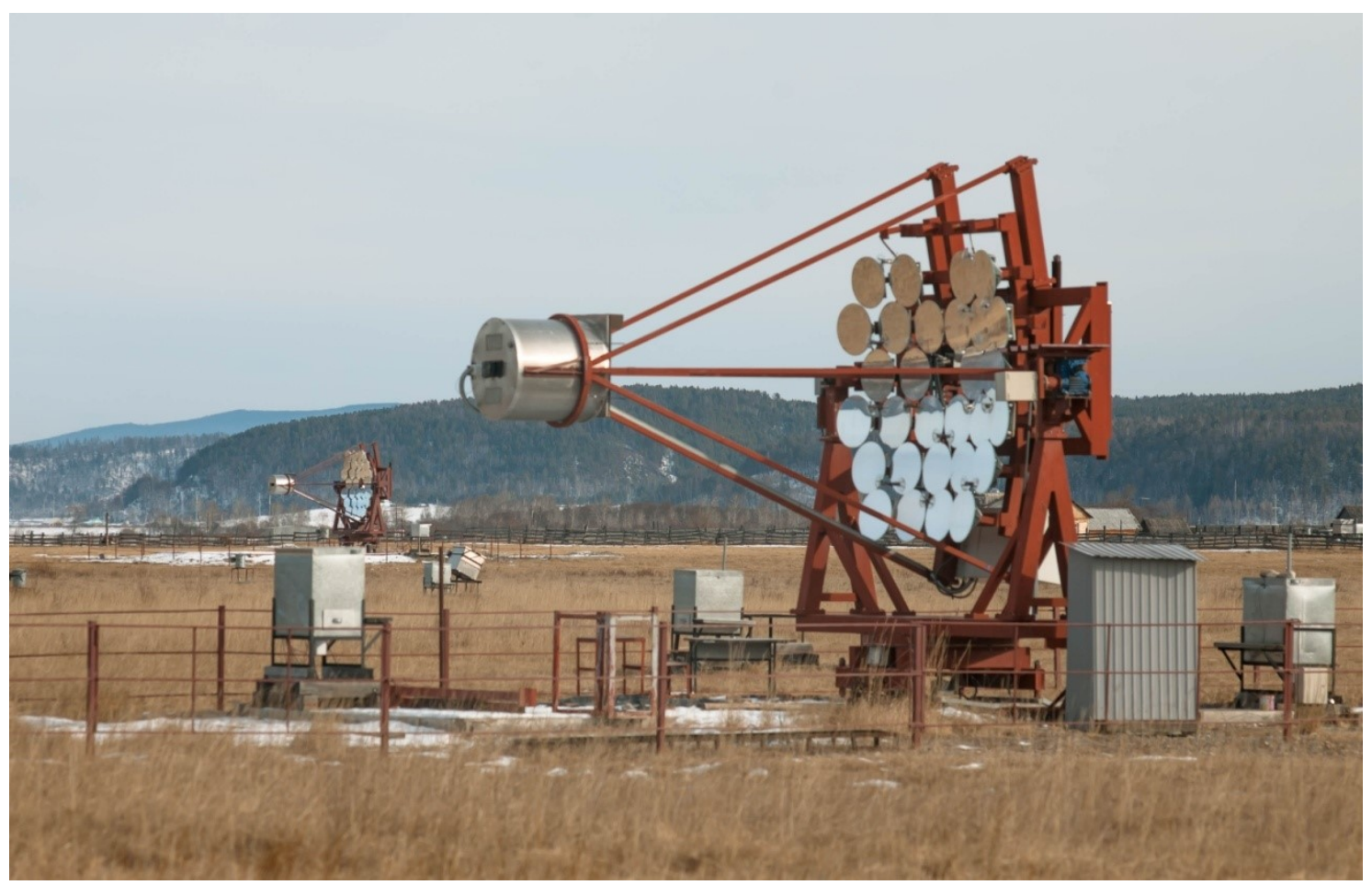

Figure 1: First 2 IACT of TAIGA observatory

In hybrid experimental setup of TAIGA observatory distance between IACTs can be up to 600-800 m because simultaneous detection by several IACT is not required for EAS parameters reconstruction. This feature makes construction of experimental installation covering large area which is required for measurements of weak gamma-ray fluxes including PeV cosmic ray sources significantly cheaper. TAIGA is the northernmost gamma-observatory and that provides some benefits in observation of high declination sources like e.g. gamma-ray source in Tycho supernova remnant. 
A. Borodin

\section{IACT mechanical mount.}

Mechanical platform of IACT can be rotated around the horizontal axis from $-10^{\circ}$ to $95^{\circ}$ and from $0^{\circ}$ to $410^{\circ}$ around azimuth axis by 2 step motors and reductors. Drive units PhyMOTION from PhyTron in conjunction with absolute 17-bit encoder HENGSTLER AC58 provide precision of telescope direction $0.05^{\circ}$ and precision of source following $5^{\prime}$. Maximum rotation speeds are $6 \% \mathrm{~s}$ in azimuth and $2 \% \mathrm{~s}$ in declination.

There are 34 glass mirrors on each IACT with diameter $0.6 \mathrm{~m}$ and total area $\sim 10 \mathrm{~m}^{2}$. Mirrors are spherical with radius of curvature $9.5 \mathrm{~m}$ and were produced in accordance with the requirements on reflectivity as well as mechanical and temperature stability. Reflective aluminium layer with width $\sim 140 \mathrm{~nm}$ was added on the surface of the mirrors by vacuum spraying followed by protective layer of $\mathrm{SiO}_{2}$ with width $\sim 200 \mathrm{~nm}$. CCD camera was attached in the center of mirror assembly for IACT direction calibration compared to known star positions.

\section{Measurements of produced IACT mechanical frame.}

\subsection{Support platforms for mirror attachment.}

Each of the 34 mirrors is attached to its own support platform on the telescope frame and rotations of those platforms should give first approximation of mirror rotations. Measurements of normal vectors to those platforms are shown on Fig.2. Telescope design assumed that those points would intersect at 0 at double focal distance and repeat mirror arrangement in 1:2 scale at single focal distance. The points are within tolerance range of mirror calibration mechanisms.

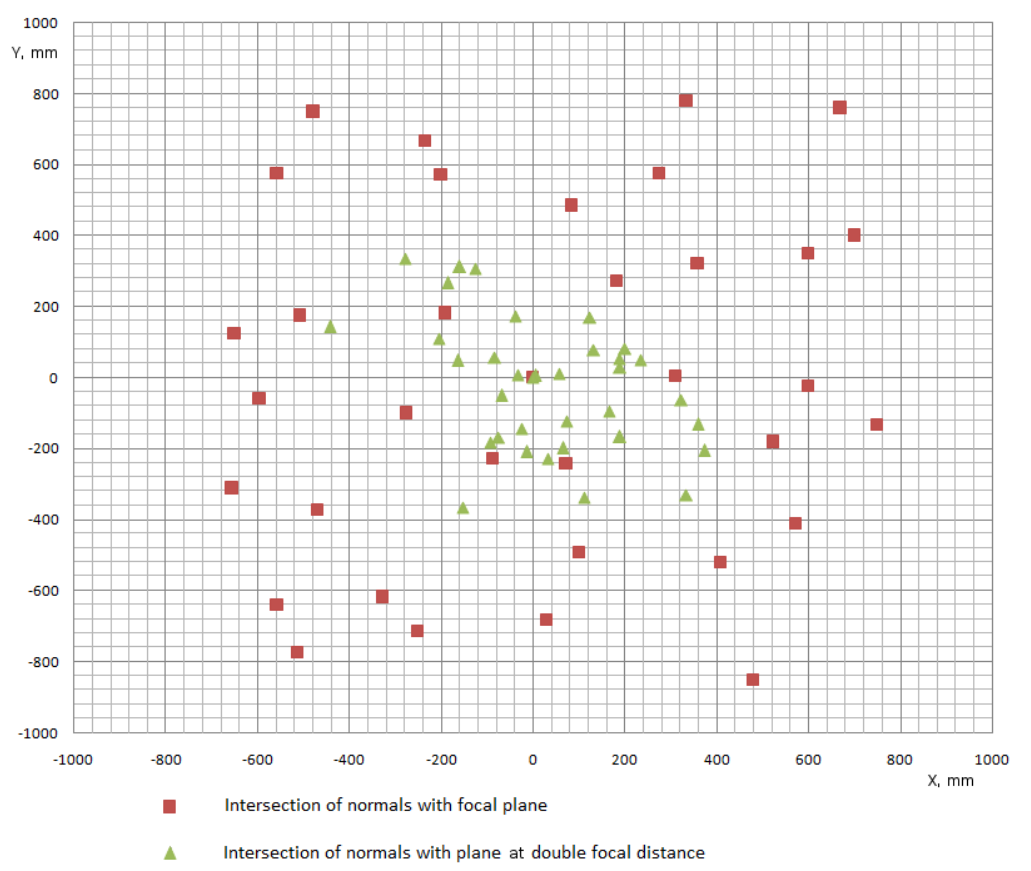

Figure 2: Measurements of intersections of vectors normal to the mirror attachment platforms with focal plane and plane at double focal distance. 
Position of support platforms was also checked. With mirror support structure in horizontal position distance from center of each support platform to a horizontal plane was measured. Difference between the measured distances and theoretical distances lies in the range from $+10 \mathrm{~mm}$ to $-5 \mathrm{~mm}$. Effectively this can be seen as changes to the distances from the focal plane to the mirrors relative to the telescope design.

\subsection{Misalignment of horizontal rotational axis.}

Digital displacement indicators were attached at both ends of rotational axis for horizontal and vertical displacement. Value of displacements were read out for different telescope rotations around the horizontal axis.

Since real telescope construction is not completely rigid, indicator values show elastic deformation of rotation axis of the telescope.

Fig. 3 shows residual difference between axis displacements and simple theoretical approximation of those displacements at the reduction gear side. Fig.4 shows the same but at the opposite side of the encoder.

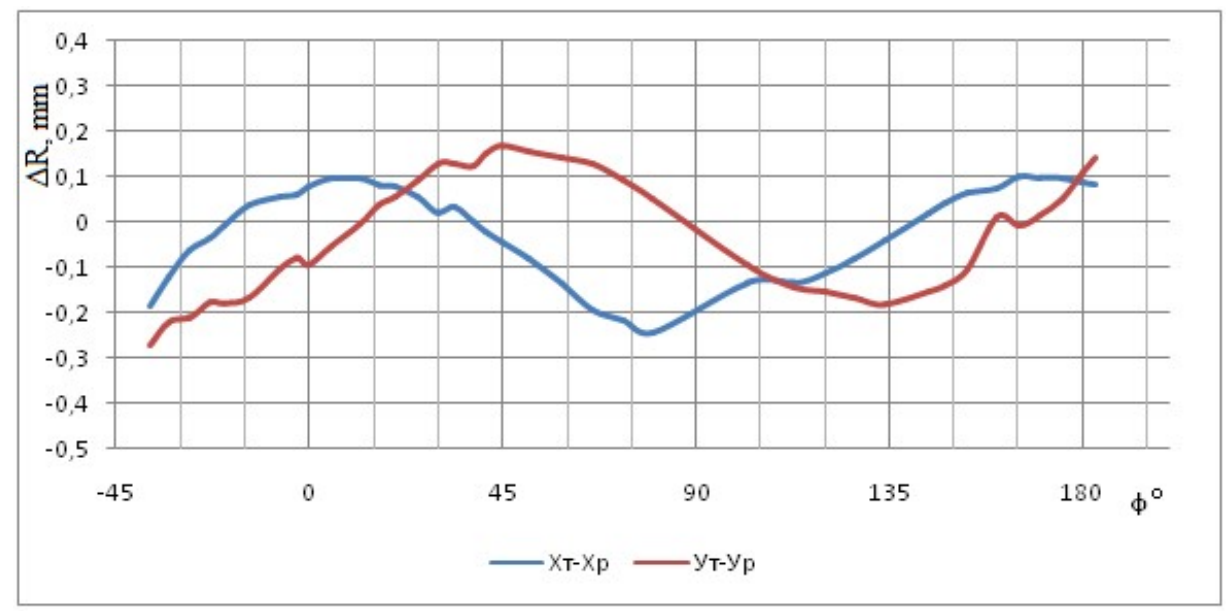

Figure 3: Difference between theoretical and practical displacements of axis at reduction gear side.

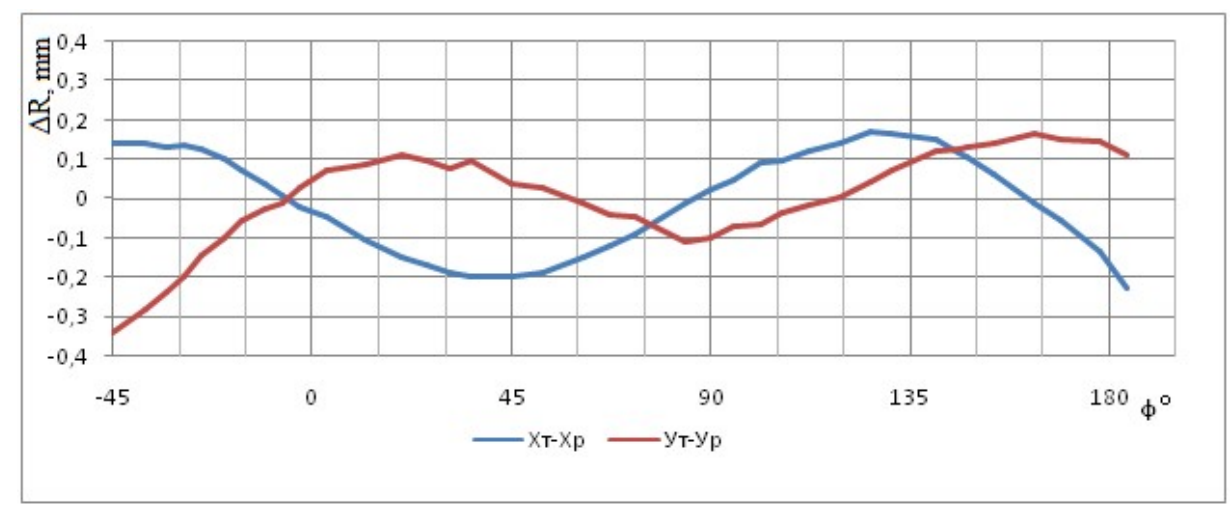

Figure 4: Difference between theoretical and practical displacements of axis at encoder side. 
Images show that residual deformations of horizontal rotation axis are within $0.5 \mathrm{~mm}$ at maximum amplitude.

Differences in the form of residual deformations graphs at encoder and reduction gear sides are explained by the details of the telescope structure: axis from the reduction gear is under stress from the torque while the axis on encoder side rotates freely and is free of such moment of force.

\subsection{Backlash of horizontal and vertical axis.}

During the backlash measurements weights were attached to telescope frame simulating weight of the camera and of the mirrors. Several series of measurements were conducted according to following procedure. Telescope was initially set horizontally, axis was lifted by $10^{\circ}$, wait for 5 seconds, lowered by $5^{\circ}$, wait 5 seconds, then repeat until the telescope is pointed to zenith. Value of backlash was measured (Fig.5) to be within $0.03^{\circ}$, which meets technical requirements of the telescope.

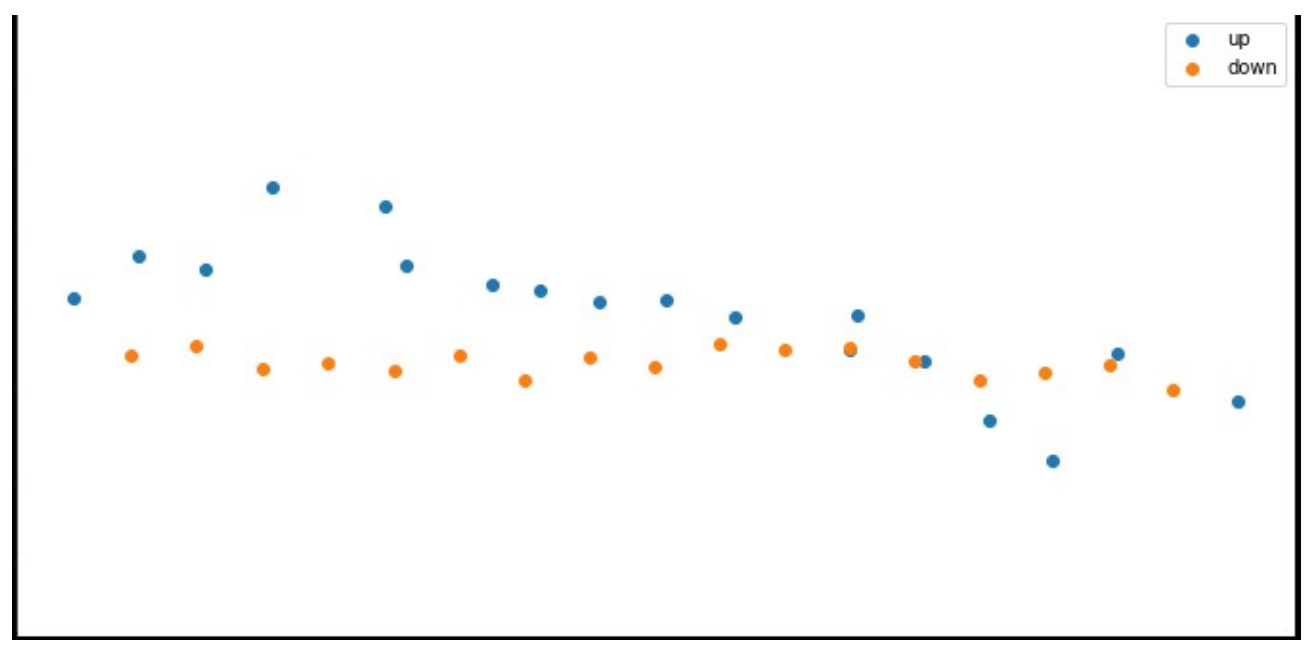

Figure 5: Measured telescope backlash during rotation around horizontal axis.

Backlash around vertical axis is reduced by additional weights with ropes and pulleys. Field operational anti-backlash system of vertical axis allows telescope rotation by $\pm 210^{\circ}$. Measurements were conducted with special system that can only rotated by $10^{\circ}$.

As can be seen on Fig. 6 weights $11 \mathrm{~kg}$ and $33 \mathrm{~kg}$ in anti-backlash system leads to backlash of $\sim 0.4^{\circ}$. Weights of $55 \mathrm{~kg}$ and more lead to backlash of $\sim 0.08^{\circ}$ which is caused by irremovable backlash of the planetary reduction gear.

\subsection{Rigidity of telescope frame.}

An optical laser was attached to mirror supports and pointed to the center of the camera. A photo camera with detached lens was placed in the center of the telescope camera such that the laser beam was shining directly at the matrix of the photo camera.

Measurements were conducted at different positions of the zenith axis of the telescope, from $-30^{\circ}$ to vertical position $\left(90^{\circ}\right)$ with a step of $10^{\circ}$ in both up and down directions. Lest side of Fig.7 shows the results of those measurements in the vertical direction with "second measurement" done 


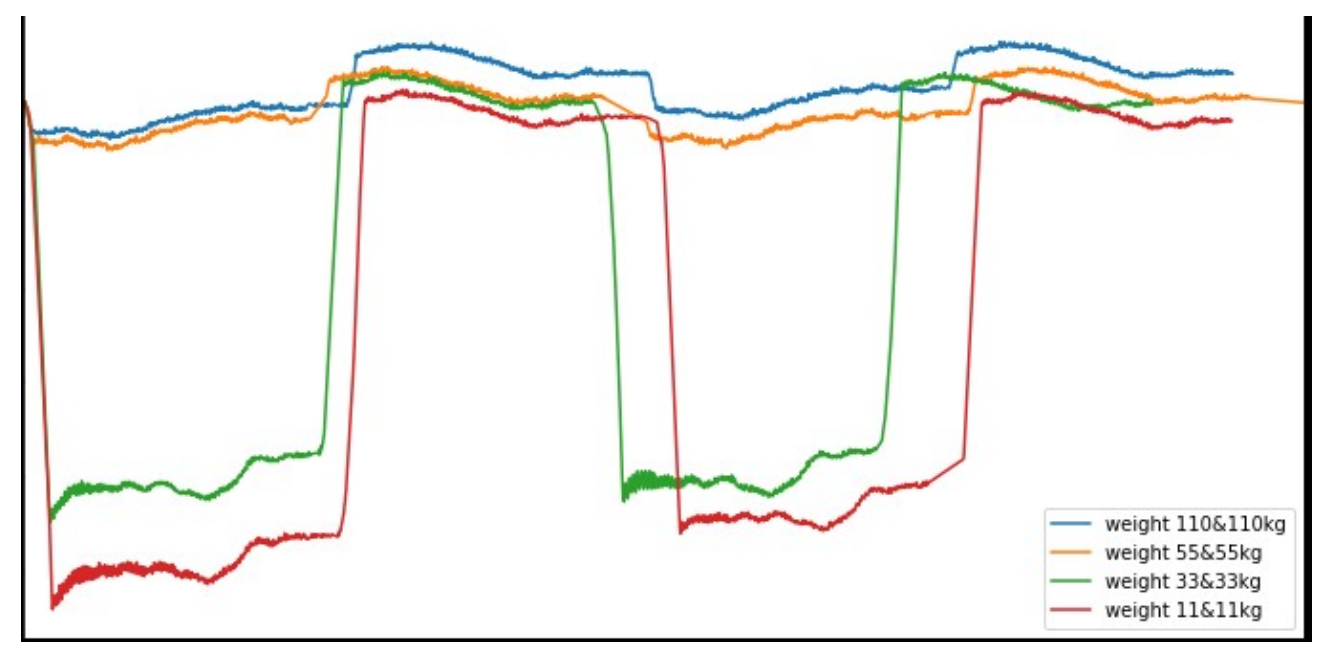

Figure 6: Measured telescope backlash during rotation around vertical axis.

after the bolts that hold the rods of the telescope camera mount were re-tightened. Right side of Fig.7 shows the same measurements but displacements in horizontal direction which have much smaller amplitude.

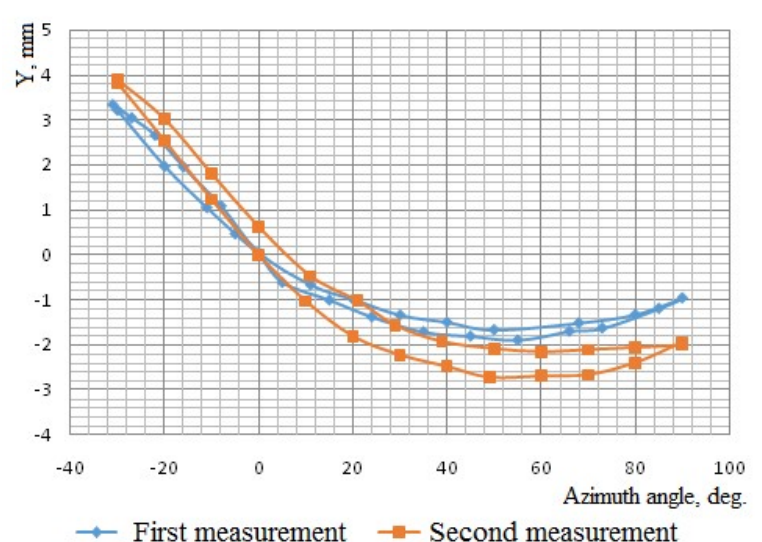

$\rightarrow$ First measurement $\rightarrow-$ Second measurement

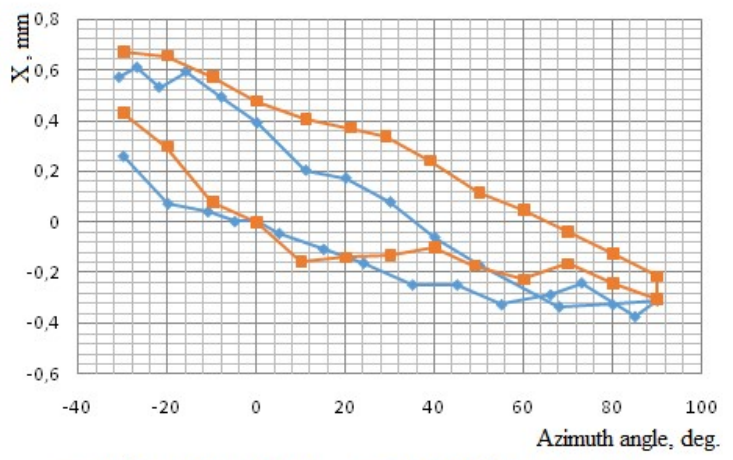

$\rightarrow$ First measurement $\rightarrow-$ Second measurement

Figure 7: (left) Displacement of the telescope camera in the vertical direction. (right) Displacement of the telescope camera in the horizontal direction.

\subsection{Static tests of the telescope.}

Direct measurements were carried out by the signal from the encoders on the vertical and on the horizontal axis of the telescope. Additional relative measurements were carried out with the use of displacements of laser beam relative to certain points at the telescope frame. Rotation angles of different parts of the telescope under load of weights simulating weight of the camera and mirrors were calculated based on those displacements.

Horizontal tangential load leads to elastic deformation of vertical axis such that the bottom part of it (place where encoder it attached) is rotating by $0.1825^{\circ}$ and upper part of the axis corresponding 
to rotation of the main telescope structure is rotated by $0.3853^{\circ}$. Accordingly the difference between encoder of vertical axis and position of main part of the telescope can take values up to $0.2^{\circ}$.

Full statical deformation from the wight of the telescope camera is $1.04^{\circ}$ according to encoder of horizontal axis. Deformation of support bracket below the reduction gear is $0.11^{\circ}$ according to optical measurements. Deformation of vertical rack is $0.08^{\circ}$. Deformation distributed along the telescope cradle is $0.93^{\circ}$.

Horizontal rotation of telescope cradle under weight load was consistent with 0 within the accuracy of the measurements.

\subsection{Natural frequencies of the telescope.}

Measurements of natural frequencies were carried out with acceleration detector of a smartphone attached to different points of the telescope frame: at the telescope camera position and at the vertical rack at the reduction gear side.

Oscillations at all frequencies in the frame of the telescope were induced with and impulse excitation. Right after that oscillograms of accelerations along all 3 dimensions were collected and later analysed. Frequency of data sets was $200 \mathrm{~Hz}$, length up to 1 minute, maximum registered value of acceleration $39.2 \mathrm{~m} / \mathrm{s}^{2}$, resolution $0.002 \mathrm{~m} / \mathrm{s}^{2}$.

Fourier analysis of accelerations detected peaks at frequencies $0.6 \mathrm{~Hz}, 2.5 \mathrm{~Hz}$ and $8.5 \mathrm{~Hz}$. Quality factors ( $\mathrm{Q}$ factors) of telescope frame at all detected natural frequencies were found by fitting the data. At $8.5 \mathrm{~Hz}$ frequency $\mathrm{Q}$ factor is $\sim 500$, at $2.5 \mathrm{~Hz} \sim 50$ and below $2.5 \mathrm{~Hz}$ it is less than 5 which corresponds to telescope structure oscillations dampening in less than 2 oscillation cycles.

\section{References}

[1] G. Pühlhofer, O. Bolz, N. Göttingetal. (The HEGRA Collaboration) The Technical Performance of the HEGRA System of Imaging Air Cherenkov Telescopes //Astroparticle Physics 2003. V.20. P. 267-291 DOI: 10.1016/j.astropartphys.2003.06.001 Preprint: astro$\mathrm{ph} / 0306123$.

[2] F.Aharonian (HESS Collaboration)//Astroparticle Physics 2011. V.34. P.738-747 HESShttp://www.mpi-hd.mpg.de/hfm/HESS/HESS.html.

[3] Albert et al // Astrophys.J.639:761-765, 2006 e-Print: astro-ph/0508543 MAGIC http://wwwmagic.mppmu.mpg.de/.

[4] V.Acciari (VERITAS Collaboaration) Ap.J Letters 730 (2011) L20 VERITAS http://veritas.sao.arizona.edu.

[5] N M Budnev, I IAstapov, A G Bogdanov, V Boreyko et al TAIGA the Tunka Advanced Instrument for cosmic ray physics and Gamma Astronomy - present status and perspectives. //Journal of Instrumentation 2014. V.9. P.C09021. doi:10.1088/1748-0221/9/09/C09021. 


\section{Full Authors List: TAIGA Collaboration}

Note comment afterwards: Collaborations have the possibility to provide an authors list in xml format which will be used while generating the DOI entries making the full authors list searchable in databases like Inspire HEP. For instructions please go to icrc2021.desy.de/proceedings or contact us undericrc2021proc@desy.de.

I. Astapov ${ }^{3}$ P. Bezyazeekov ${ }^{1}$ A. Borodin ${ }^{8}$ M. Brueckner ${ }^{5}$ N. Budnev ${ }^{1}$ A. Chiavassa ${ }^{12}$ A. Dyachok ${ }^{1}$ O. Fedorov ${ }^{1}$ A. Gafarov ${ }^{1}$ A. Garmash ${ }^{10,11}$ V. Grebenyuk ${ }^{8}$ O. Gress ${ }^{1}$ T. Gress ${ }^{1}$ A. Grinyuk ${ }^{8}$ O. Grishin ${ }^{1}$ D. Horns ${ }^{6}$ A. Ivanova ${ }^{1}$ N. Kalmykov ${ }^{4}$ Y. Kazarina $^{1}$ V. Kindin ${ }^{3}$ P. Kirilenko ${ }^{10}$ S. Kiryushin ${ }^{1}$ R. Kokoulin ${ }^{3}$ K. Kompaniets ${ }^{3}$ E. Korosteleva ${ }^{4}$ V. Kozhin ${ }^{4}$ E. Kravchenko ${ }^{10,11}$ M. Kunnas ${ }^{6}$ L. Kuzmichev ${ }^{4}$ Yu. Lemeshev ${ }^{1}$ V. Lenok ${ }^{15}$ N. Lubsandorzhiev ${ }^{4}$ B. Lubsandorzhiev ${ }^{7}$ R. Mirgazov ${ }^{1}$ R. Mirzoyan ${ }^{2}$ R. Monkhoev ${ }^{1}$ R. Nachtigall $^{6}$ E. Osipova ${ }^{4}$ A. Pakhorukov ${ }^{1}$ A. Pan $^{8}$ M. Panasyuk ${ }^{4}$ L. Pankov ${ }^{1}$ A. Petrukhin ${ }^{3}$ V. Poleschuk ${ }^{1}$ E. Popescu ${ }^{13}$ E. Popova ${ }^{4}$ A.

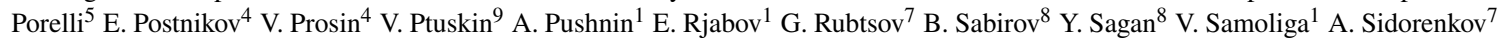
A. Silaev ${ }^{4}$ A. Silaev (junior) ${ }^{4}$ A. Skurikhin ${ }^{4}$ V. Slunechka ${ }^{8}$ A. Sokolov ${ }^{10,11}{ }^{11}$ Y. Suvorkin ${ }^{1}$ L. Sveshnikova ${ }^{4}$ V. Tabolenko ${ }^{1}$ A. Tanaev ${ }^{1}$ B. Tarashansky ${ }^{1}$ M. Tluczykont ${ }^{6}$ L. Tkachev ${ }^{8}$ R. Wischnewski ${ }^{5}$ I. Yashin ${ }^{3}$ A. Zagorodnikov ${ }^{1}$ and D. Zhurov ${ }^{1}$

${ }^{1}$ Applied Physics Institute of Irkutsk State University, Karl Marx street, 1, 664003 Irkutsk, Russian Federation. ${ }^{2}$ Max-Planck-Institute for Physics, Föhringer Ring 6, 80805 Munich, Germany. ${ }^{3}$ National research nuclear university "MEPhI”, Kashirskoe shosse, 31, 115409 Moscow, Russian Federation. ${ }^{4}$ Skobeltsyn Institute of Nuclear Physics of Moscow State University, Leninskie gory 1, building 2, 119991 Moscow, Russian Federation. ${ }^{5}$ Deutsches Elektronen-Synchrotron DESY, Zeuthen, Platanenallee 6, 15738 Zeuthen, Germany. ${ }^{6}$ Institute for Experimental Physics, University of Hamburg, Mittelweg 177, 20148 Hamburg, Germany. ${ }^{7}$ Institute for Nuclear Research, Prospekt 60-let Oktyabrya 7a, 117312 Moscow, B-312, Russian Federation. ${ }^{8}$ Joint Institute for Nuclear Research, Joliot-Curie 6, 141980 Dubna, Moscow region, Russian Federation. ${ }^{9}$ Institute of Earth magnetism, ionosphere and radiowaves propagation named after Nikolay Pushkov, Troitsk, Kaluzhskoe Hwy 4, 142190 Moscow, Russian Federation. ${ }^{10}$ Novosibirsk State Universit, 1, Pirogova str., Novosibirsk, 630090, Russian Federation. ${ }^{11}$ Budker Institute of Nuclear Physics of Siberian Branch Russian Academy of Sciences, 11, Acad. Lavrentieva Pr., Novosibirsk, 630090 Russian Federation. ${ }^{12}$ Università degli Studi di Torino, Via P. Giuria, 1 - 10125 Torino, Italy. ${ }^{13}$ Institute of Space Science, 409, Atomistilor Street, Magurele, Ilfov, Romania, 077125. ${ }^{14}$ Altai State University, pr. Lenina 61a, Barnaul, 656049, Russian Federation. ${ }^{15}$ Karlsruhe Institute of Technology (Guest Institute Tunka-REX), P.O. Box 3640, 76021 Karlsruhe, Germany. 